\title{
Quick Routing in Autonomous Vehicles using Boosting Algorithms
}

\author{
Prem Raja D, Vasudevan V
}

\begin{abstract}
Self-driving automobiles are understandably the most attention grabbing utility of artificial intelligence. Until recently, we have just considered the prototypes of these cars in Sci-fi movies, with the whole thing else left to our imagination. But with advances in technology, this super notion has acquired a lifestyles of its own. Autonomous vehicle promises to improve traffic safety while at the same time, it must increase the fuel efficiency, reduce congestion and arrive to the destination at a minimum time span. We propose a novel technique to boost the algorithm to take the shortest path while the vehicle is in movement.
\end{abstract}

Keywords : Autonomous vehicle, fuel efficiency, congestion, boost

\section{INTRODUCTION}

Autonomous cars are expected to play a key function in the future of city transportation systems, as they supply attainable for more safety, improved productivity, larger accessibility, better street effectivity and remarkable impact on the surroundings. Determination of suitable path for autonomous vehicles that is collision free between initial and end position through the path in the presence of obstacle is challenging for autonomous vehicle design.

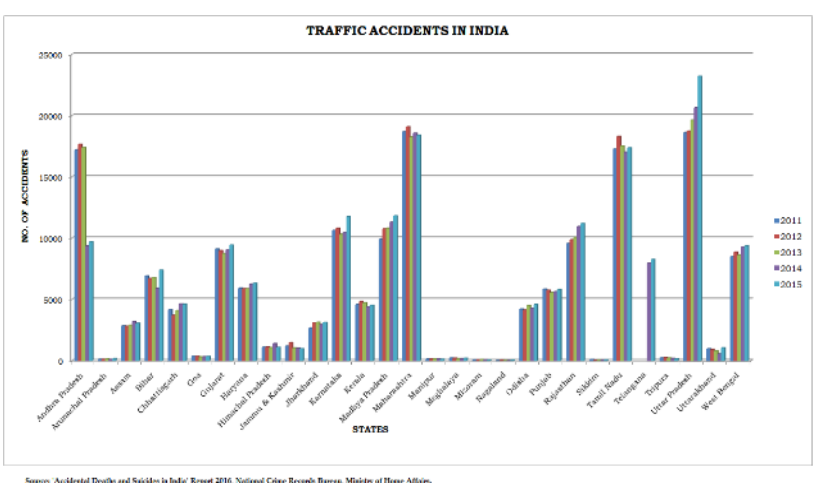

Fig.1:Road Accidents in different States in India

Indian authorities has released the reliable information for road accidents, accidents and fatalities for the year 2017 (fig. 1), (fig.2) and the information continues to be bad. A total of 4 lac road accidents have been stated in the country,

Revised Manuscript Received on December 16, 2019.

* Correspondence Author

Prem Raja D*, Department of information Technology, Kalasalingam Academy of Research and Education,Tamil Nadu, INDIA. Email: dpremraja@gmail.com

Vasudevan V, Department of information Technology, Kalasalingam Academy of Research and Education,Tamil Nadu, INDIA. Email: vasudevan_klu@yahoo.co.in claiming greater than 1 lac lives and inflicting accidents to greater than 4lac persons. In the year 2018 over eight lac of humans died in road accidents due to rash driving, drunken drive, violating traffic regulations etc.

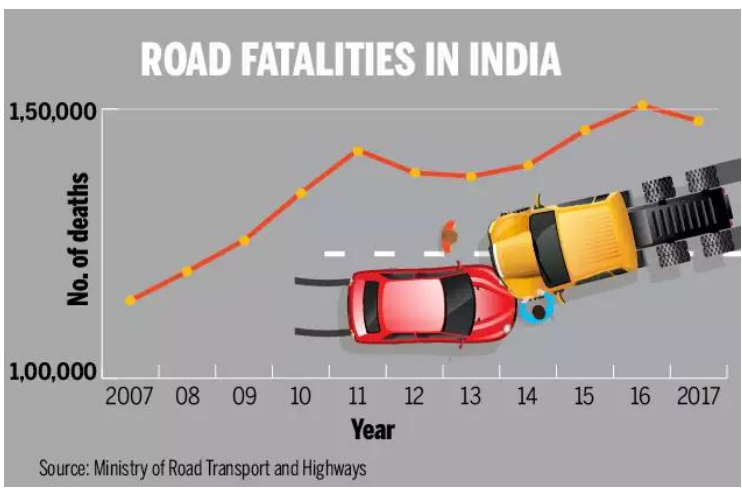

Fig.2: Road Fatalities in India

Youth the largest causality of road crashes

Its formative years lives that are suffered out the most due to road accidents in India. In 2016 (fig.4), a impressive 60\% of human beings who lost their lives in street accidents were in the age of 18-35 years (fig.3). Tragically, majority of them had been the sole incomes member of their family.

\section{ROAD DEATHS BY AGE-GROUP}

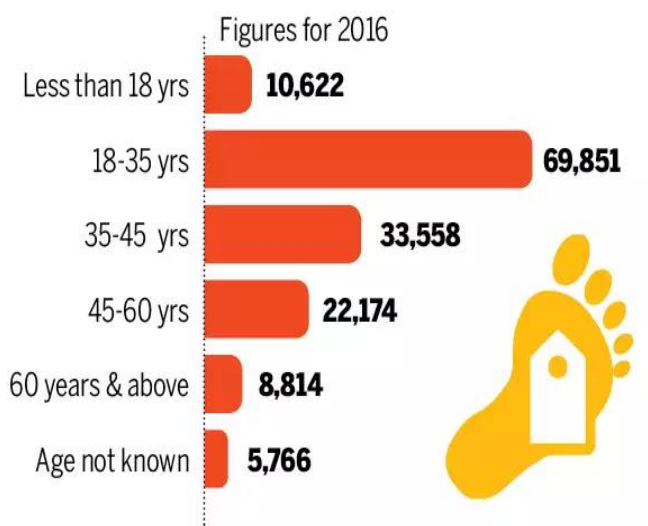

Source: Ministry of Road Transport and Highways

Fig.3:Statisitics on death by age group 


\section{OVERSPEEDING THE LEADING KILLER ON INDIAN ROADS}

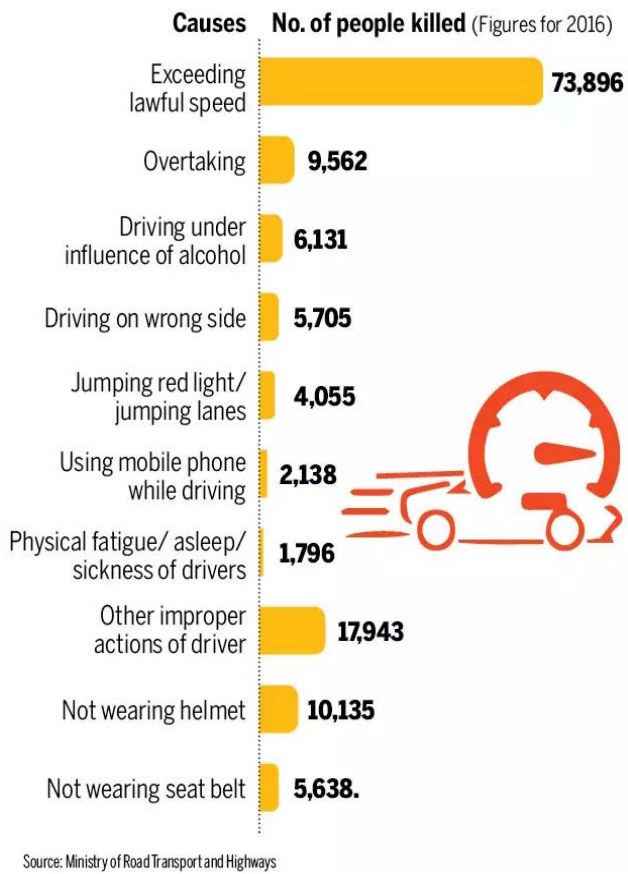

Fig.4:Statisitics on over speeding

\section{In-efficient Enforcement}

To decrease accidents, the regulation needs to be enforced properly. As per WHO, wearing seat belts reduces the threat of a deadly harm among the front seat passengers by way of up to $50 \%$ and for the rear seat passengers up to $75 \%$ (fig.5).

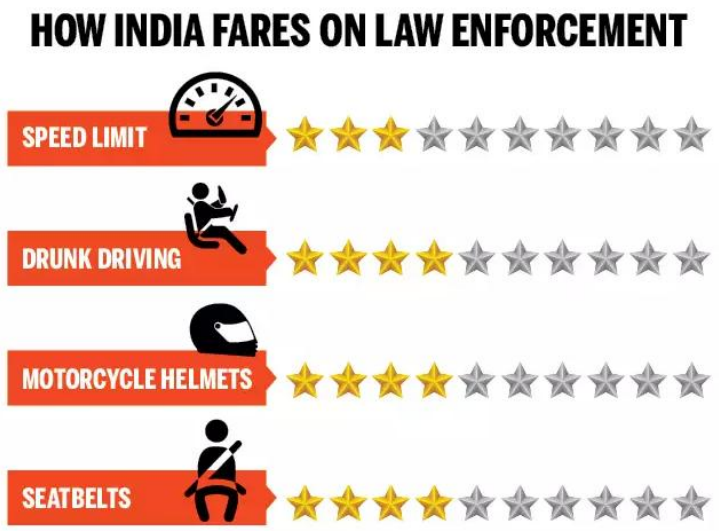

Source: WHO Global Status Road Safety Report 2015

Fig.5:Statisitics on over speeding

\section{Ada Boosting}

Ada boosting or Adaptive boosting is a aggregate of more than one getting to know algorithms that can be utilized for regression or classification. It overcomes overfitting when compared with any different computing device getting to know algorithms and is regularly sensitive to noisy data. In order to create one composite effective learner Ada boost makes use of multiple iterations. So it is termed as adaptive. By adding susceptible newbies iteratively, Ada raise creates a robust learner i.e., Ada improve helps in boosting the weak threshold classifier to strong classifier ${ }^{[11]}$.

\section{XG Boost}

It is a open supply software library which affords a gradient boosting framework. It ambitions to furnish a scalable, portable and distributed gradient boosting. Other than strolling on a single machine, it additionally supports the disbursed processing framework.

\section{RELATED WORK}

A soft-cascade Ada boost classifier with Haar features ${ }^{[1]}$ is trained for vehicle recognition and applied to images to provide frontal and rear vehicle detection. The algorithm which is used takes much time to resolve the image as there is noise captured in the images of the camera.

The Ada boost getting to know method ${ }^{[2]}$ for vehicles detection with a single moving camera used to be proposed . the global symmetric features and nearby symmetric facets are described and used to assemble a cascade structure. The Ada boost studying algorithm is used for the education process.

Boosting has been a very successful technique for solving the two type classification problems with their Ada boosting algorithm. When shifting from two category to multi category classification, most boosting algorithms have been confined to lowering the multiclass classification to multiple two class classification $^{[3]}$.

Detecting large animals on roadways using automated systems is a vital task. This can be used by conventional tools such as ultrasonic sensors, smart cameras etc., ${ }^{[4]}$. The boosting of algorithms to detect the surrounding of the vehicle using online Ada boost approach ${ }^{[5]}$.

Autonomous automobiles are substantially anticipated to alleviate road congestion with the aid of higher throughput, improve road protection by using way of putting off human error and free drivers from the burden of driving ${ }^{[6]}$.

Autonomous vehicle systems combine various techniques for perceiving the environment using popular sensors and algorithms ${ }^{[7]}$. Methods that solve the specific tasks use analytical algorithms as well as methods of artificial intelligence which in some case produce much better result ${ }^{[8]}$

Fully automated cars will enable drivers to be pushed through an informatics system in their own vehicle, which helps the drivers to engage in non-driving related activities ${ }^{[9]}$. A stimulator for the lane maintaining device is constructed the usage of image projection, automobile trajectory tracking ${ }^{[10]}$.

\section{PROPOSED WORK}

The proposed system uses a novel technique in boosting the algorithm to find the better path to make the autonomous vehicle move from source to destination without any hindrances and in an optimal time span.

\section{Reinforcement Learning}

Reinforcement studying is a education method primarily based on profitable favored behaviours and / or punishing undesired ones. The getting to know approach has been adopted in artificial Genius as a method of directing unsupervised computing device learning thru reward and penalties. Reinforcement is used in operation research, statistics theory, recreation theory, manage theory, simulation-based optimization, multi agent systems, swarm intelligence, data and genetic

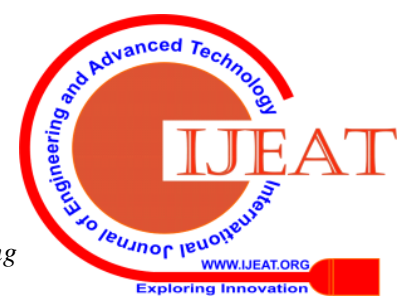


algorithms.

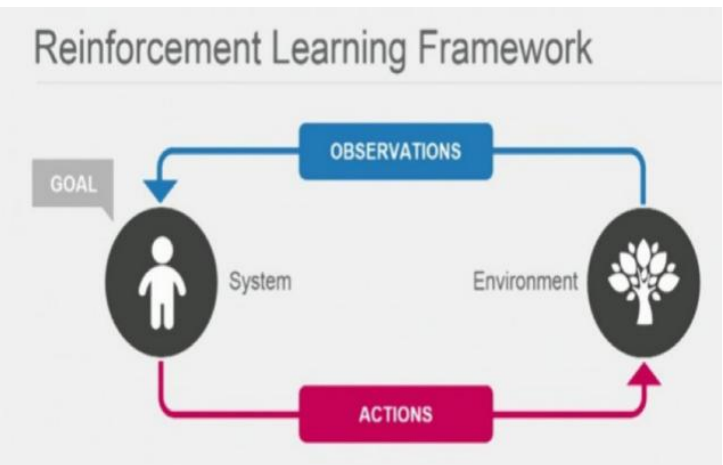

Fig.6:Reinforcement Learning Framework

\section{Ensemble}

Ensemble is a machine learning technique that combines several base models in order to produce one optimal predictive model.

\section{Types of Ensemble Methods}

$>$ BAGGing - This gets its name because it combines Bootstrapping and AGGregration to from one ensemble model.

$>$ Random Forest - The Random Forest model decide where to split based on a random selection of features. Rather than splitting at similar features at each node throughout.

The ensemble that is created uses both the BAGGing and Ramdom Forest method to attain to the conclusion that to which path to be selected in a minimum time span and provide the instruction to the autonomous vehicle and make the vehicle move.

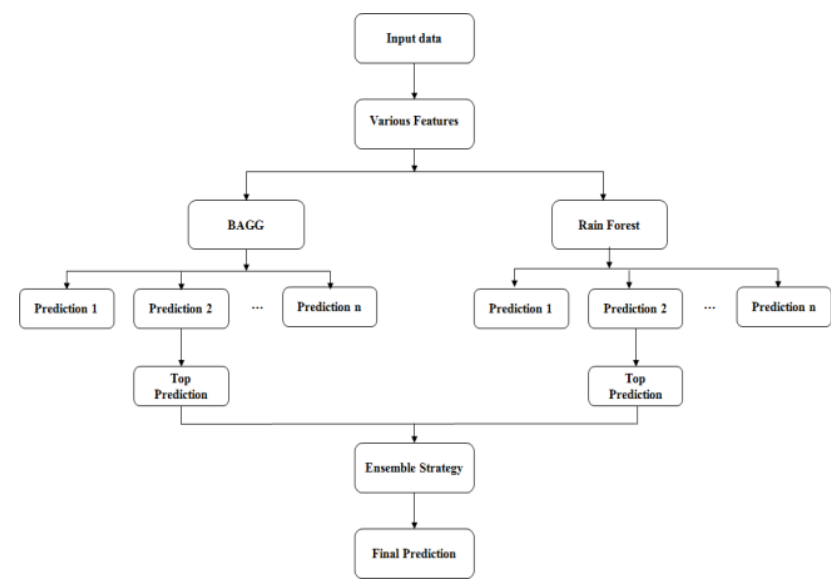

Fig.7:Proposed Model

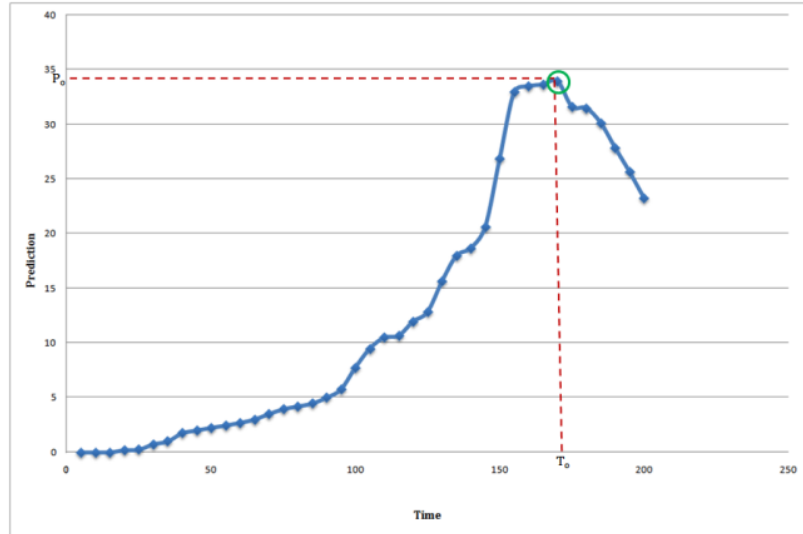

Fig8:Bagging Technique

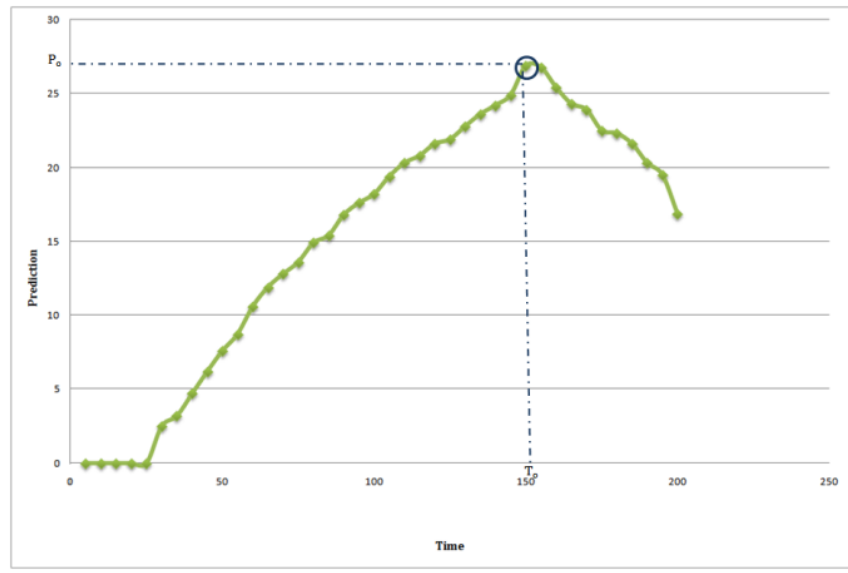

Fig.9:Random Forest Technique

Taking the top most prediction from both the techniques and implementing in the proposed algorithm will give us the final prediction and that will be an accurate prediction.

\section{CONCLUSION AND FUTURE WORK}

In this paper we proposed a technique that makes the autonomous vehicle to act fast based on the surrounding of the vehicle and make the correct decision to take the right path and move the autonomous vehicle from the start to the end of the journey. In future an artificial intelligence system will be implemented inside the vehicle which will monitor the inside condition of the passenger such as health condition, and the ventilation of air inside the vehicle. If there is any emergency in the case of the health issues to the passenger the system will automatically intimate the situation to the nearest hospital, fire station and police station ad re-route the path to the nearest hospital.

\section{ACKNOWLEDGMENT}

The authors would like to thank the Department of Information Technology of Kalasalingam Academy of Research and Education, Tamilnadu, India for permitting to use the computational facilities available in open source research laboratory.

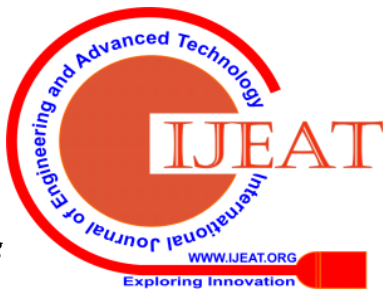




\section{REFERENCES}

1. Broggi et. al. "Vehicle Detection for Autonomous Parking usng Soft-Cascade AdaBoost Classifier" June 8-11, 2014 IEEE Intelligent Vehicles

2. Tie Liu, Nanniang Zheng, Li Zhao, Hong Cheng "Learning based Symmetric Features Selection for Vehicle Detection" 2005 IEEE

3. Ji Zhu, Hui Zou, Saharon Rosset and Trevor Hastie "Multi-class AdaBoost" Statistics and Its Interface 2009

4. Abdelhamid Mammeri, Depu Zhou and Azzedine Boukerche "Animal-Vehicle Collision Mitigation System for Automated Vehicles" 2016 IEEE Transactions on Systems, Man and Cybernetics: Systems

5. Wen-Chung Chang and Chih-Wei Cho "Online Boosting for Vehicle Detection" June 2010 IEEE Transactions on Systems, Man and Cybernetics

6. Scott Drew Pendleton et. al. "Perception, Planning, Control and Coordination for Autonomous Vehickes" www.mdpi.com/journal/machines, Machines 2017,5,6; doi:10.3390/machines5010006

7. Abhishek Nayak, Swaminathan Goplswamy and Sivakumar Rathinam "Vision-Based Techniques for identifying Emergency Vehicles" SAE International 02 April 2019

8. Michal Bugala "Algorithms applied in Autonomous Vehicle Systems" research gate publication December 2018

9. Dr. K. Velmurugan et. al. "Automated Vehicle: Autonomous Driving using SVM Algorithm in Supervised Learning" IJERT RTICCT 2019

10. Zhilu Chen "Computer Vision and Machine Learning for Autonomous Vehicles"

11. https://www.kdnuggets.com/2017/06/machine-learning-algorithms-us ed-self-driving-cars.html

\section{AUTHORS PROFILE}

Prem Raja he is a working as a Assistant professor and a Part-Time research scholar in the department of Information Technology at Kalasalingam Academy of Research and Education, Krishnankoil, Srivilliputhur India. His areas of interest are big data analytics, autonomous vehicles and security.

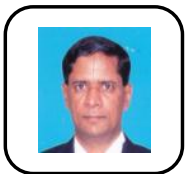

Vasudevan he is working as senior professor in the department of information technology of Kalasalingam Academy of Research and Education Krishnankoil Srivilliputhur India for past 27 years and his areas of interest are big data analytics, cloud computing, network security and block chain technology. He has published 170 above papers in international journals and conferences. He has produced twenty above $\mathrm{PhD}$ scholars and currently guiding five PhD scholars. He is a life time member in ISTE and IAENG. He received Dr. APJ Abdul Kalam Award for life time contribution in teaching on 2016. 